\title{
Urgences
}

\section{Jacques Daignault, Pour une esthétique de la pédagogie , Victoriaville, NHP, 1985, 260 p.}

\section{Thérèse Paquin}

Numéro 16, mars 1987

D.G. Jones : d'un texte, d'autres

URI : https://id.erudit.org/iderudit/025412ar

DOI : https://doi.org/10.7202/025412ar

Aller au sommaire du numéro

\section{Éditeur(s)}

Urgences

\section{ISSN}

0226-9554 (imprimé)

1927-3924 (numérique)

Découvrir la revue

Citer ce compte rendu

Paquin, T. (1987). Compte rendu de [Jacques Daignault, Pour une esthétique de la pédagogie , Victoriaville, NHP, 1985, 260 p.] Urgences, (16), 102-104.

https://doi.org/10.7202/025412ar d'utilisation que vous pouvez consulter en ligne.

https://apropos.erudit.org/fr/usagers/politique-dutilisation/ 


\section{deliveline}

\section{Jacques Daignault: Pour une esthétique de la pédagogie, Victoria- ville, NHP, 1985, 260 p.}

\section{Un scandale... à suivre}

Voilà un ouvrage aux desseins bien ambitieux, voire utopiques: racheter une erreur de l'histoire de la pensée et donc de la mission pédagogique, en faisant de la pédagogie un art et du pédagogue un artiste. Les hypothèses qui le gouvernent sont fondées sur des modèles théoriques déjà bien étayés dans certains ouvrages philosophiques auxquels l'auteur fait appel. C'est un livre qui prolifère parfois de toutes parts, cherchant plus à réaliser la mise en scène théâtrale que la cohérence. Mais là n'est pas le mal puisque, dans l'introduction, l'auteur se targue d'en faire, outre un ouvrage philosophique, «un roman pédagogique, un genre qui se développe à travers l'argumentation avouant une préférence: le style devant l'emporter sur la signification».

Pour qui a pratiqué Deleuze et Lyotard, l'impression première est que ça frôle le pastiche. On se rend vite compte que l'on est devant cet ouvrage universitaire, espèce de «readings» oũ tous les points de vue sur un sujet sont refondus par un commentateur savant. L'impression perdure malgré les aveux fréquents d'emprunt et les nombreuses références. Peut-être cela est-il dû à l'ardeur immodeste de la jeunesse grisée par sa propre habileté à renouveler les exploits de ses maîtres à penser, toute heureuse de se glisser dans l'enceinte sacrée des gardiens du pouvoir inhérent au savoir du langage. Il ne faut donc pas oublier que l'auteur en est à ses premières armes philosophico-littéraires, cet essai étant sa thèse doctorale légèrement remaniée, du moins je crois.

Je tâcherai de faire la lumière sur les principaux éléments de l'hypothèse proposée ici sans tenir compte de tous les déploiements théoriques qui en constituent à la fois la force et la faiblesse. Force d'une certaine logique greffée en abyme à l'exposé de certaines thèses, faiblesse de la lourdeur des jeux logiques souvent trop obligés. La thèse principale proposée ici est la suivante: la transparence du savoir sur laquelle repose la pédagogie est un échec, et son seul salut réside dans la constitution d'un langage déterminé par sa nature propre.

Dans un premier volet, l'auteur se donne comme tâche de retracer la genèse de cet échec de la pédagogie objective et normative; dans un deuxième volet, il s'emploie à démontrer qu'il existe une autre voie où la pédagogie peut s'engager: celle de la "séduction" où la "médiation de pertinence» n'est plus arbitrée par le «jugement de pertinence». Tâche difficile que cette dernière, «hypothèse trop complexe pour qu'on puisse en venir à bout dans un seul commentaire à la suite des autres". L'hypothèse, dit-il, "musardera donc entre les lignes", et le lecteur aussi.

\section{Origines de l'objectif et du nor- matif}

La pédagogie n'a jamais cessé d'être une science objective et normative. Elle n'a jamais cessé non plus d'être embarrassée de cette objectivité et de cette 
normativité. C'est aux querelles opposant les Sophistes à Socrate et à Platon que l'on fait remonter le débat. Platon, philosophe scientifique dévoué à la connaissance de toutes choses, s'est empressé de refuser toute prétention au savoir universel; Socrate, philosophe de la vérité, enseigne le savoir qu'il est seul à détenir. «Virtuose de l'ironie (...) il dénoue patiemment tous les fils de la séduction (...) pour séduire à son tour dans un climat de confiance préparé par lui. Socrate devient peu à peu l'accoucheur de la vérité». Protagoras devra fournir des réponses claires à Socrate. Il devra abandonner le langage de la séduction, cesser de raconter des histoires. Voilà la scène première à laquelle feront écho toutes les discussions philosophiques subséquentes visant à définir la nature du dialogue pédagogique. L'auteur affirme aque le message légué à la postérité par Socrate et Platon offre les conditions d'un arbitrage $d u$ "sens" par la "signification", de la médiation de pertinence par le jugement de pertinence, de la relation affective par la relation d'autorité». L'appel du chant d'Orphée, l'hésitation prolongée entre le son et le sens sont remplacés par la pertinence du discours. La médiation de pertinence ou la relation pédagogique fera en sorte que ce qui n'a pas de sens puisse en prendre au terme d'un processus. Toute l'empirie du dialoque ainsi que tout tiers - le corps des mots, le corps humain lui-même - seront exclus. La définition d'une bonne éducation ne dépendra que des valeurs de ceux qui entreprennent de la définir. "La légitimité d'imposer un sens et une direction aux attitudes et aux savoirs n'aura donc jamais cessé de faire problème». Le statut du discours pédagogique ne pourra que rester indéterminé; la pédagogie, entièrement vouée à la cause du savoir, deviendra le tiers-monde du savoir. L' $\mathrm{i}$ dée de Totalité viendra renforcer celle de hiérarchie, de relation d'autorité, et cela dans un seul but: "faire triompherle règne des nécessités contre le hasard, l'arbitraire, le contingent et le désordre logique, social, affectif, politique». Paradoxe du sens rétréci, limité au terrorisme du sens commun et du bon sens.

\section{Triompher par le «scandale»}

L'auteur annonce que les solutions proposées pour sortir de la nécropole scolaire engendrée par cette longue sclérose philosophique seront de l'ordre du «scandale»: "celui d'une promesse qui ne pourra être tenue." Cette promesse sera celle de Don Juan, dont la jouissance résidera dans le fait même de promettre. Le mensonge n'existera que pour le point de vue "constatif». Il faudra risquer, comme les Sophistes, de n'avoir rien à dire, de ne parler que pour s'entendre parler, que pour évoquer le chant des sirènes, pour «séduire, donnerà jouir à propos de la connaissance». La spécificité de la narration pédagogique reposera sur «la monstruosité entre la jouissance et la connaissance, entre fiction et vérité». Elle devra créer un effet d'indécidabilité.

La victoire contre les Sophistes entraînera le découpage - bien défini par Kant - de deux espaces de l'éducation, l'un (technologique) déterminé par la rêduction entre ce "qui est» et ce qui "devrait être», l'autre (politique) déterminé par l'irréductibilité de ce même espace. S'ensuivra la prescription des objectifs et des moyens en pédagogie.

Daignault démontrera au moyen d'un récit métaphorique emprunté à Clément Rosset que les grandes fins de l'éducation sont vouées à l'échec, parce qu'elles sont de l'ordre du Désir. De cet échec résulteront le "nihilisme» ou la fuite du désir irréalisable et le «terrorisme» ou la réduction de la réalité. Devant ce bilan négatif, il restera comme alternative, pour le pédagogue, le projet de «faire savoir comme on fait rire» propre à Protagoras et à Don Juan. C'est au langage expressif qu'il faudra emprunter ce pouvoir de faire rire, puisque seul l'écrivain met en scène ce paradoxe de ne pouvoir rien ajouter aux choses par l'écriture, apportant la solution à l'échec de la désignation par la «célébration». La pédagogie, à l'instar de la littérature, devra délaisser son oeuvre de conciliation (subordination de la ficcion à la vérité) et «créer un effet 
d'indécidabilité quant au statut du "narré»». Mais il faudra encore démontrer cette thèse "par une réponse "célèbre» impliquant à la fois la phénoménologie et la paradoxologies. Pour indiquer quelle conception la phénoménologie se fait de l'espace littéraire, Daignault développe les thèmes de l'universel singulier emprunté à Sartre, de l'émergence de la subjectivité emprunté à Kant, du structuralisme différentiel et de la double négativité empruntés à Lyotard. Toutes ces intrigues sur la constitution du sens trouveront leur dénouement éclairé par une histoire et un dialogue: «Euzèbe ou l'allégorie des écarts» et "Don Juan et Don Quichotte». $\mathrm{Ne}$ faut-il pas enfin pratiquer ce que l'on propose... ou prescrit? Illustrer par la pratique textuelle cette lutte à finir avec le bon sens et le sens commun?

Thêrèse Paquin

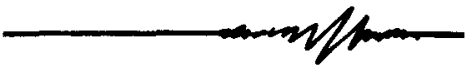

Clermont Gauthier: Une éducation juste ou juste une éducation? Critique des courants pédagogiques contemporains. Victoriaville, HNP, 1986, 268 pages.

L'ouvrage que vient de publier Clermont Gauthier est stimulant pour la réflexion, mais peut-être frustrant pour l'action. Le lecteur qui parcourt cette magnifique fabulation autorisée, philosophiquement désinfectée, et croit trouver à la fin une réponse à ses interrogations d'éducateur, se verra peut-être ramené au commencement de ses hypothèses sur les rapports théorie/pratique.

Je ne dirais pas de ce livre que "ça vaut ce que ça vaut", mais que ça vaut ce que l'auteur veut. Je crois qu'il veut troubler une certaine somnolence en se donnant le mal de faire voir le jamais vu sous le trop connu, ou le jamais trop su sous le tant appris et ainsi provoquer un questionnement neuf.
C'est un péché intellectuel en deux parties qui désillusionne par un souci d'équité et par la démonstration qu'une simple décision de bons sens ne fait pas la clarté. Il brouille ainsi les histoires qu'on se raconte et qu'on raconte sur les finalités, les moyens, les besoins ou les désirs relatifs à l'éducation.

Les plus injustes ne pourront pas accuser l'auteur de n'avoir pas courtisé l'IDÉE et ceux qui en font profession, ni de s'être pas laissé séduire par les Kant, Deleuze ou Lyotard, entre autres. C'est ainsi qu'à partir d'une mosaïque de points de vue, l'ouvrage s'organise, dans la première partie, autour des trois dimensions du discours éducatif: science, éthique et technique mises $\in \mathrm{n}$ relation.

Chacune de ces dimensions étant un donné théorie/pratique, la pratique étant assimilée au réel et la théorie à la représentation du réel, la pratique oblige la théorie à l'exactitude, et la théorie guide la pratique. La théorie s'entendant ici comme points de vue ou "simulacres", soumis à des tests de vérité, dépasse lopinion. La théorie renvoie à une dimension scientifique et ainsi décrit, alors que la pratique prend une dimension éthique ou prescriptive. Est-il possible, se demande l'auteur, de combler l'écart entre ce qui est et ce qui doit être? Sinon, comment fonder ce qui doit être, le JUSTE? Comment guider un individu vers un état souhaitable, comment éduquer? Affaire de convention ou affaire de vérité?

L'éducation est posée comme une activité de désir (mieux-être impossible à combler) puisqu'elle renvoie à des finalités sans cesse questionnées, et non comme une réponse au besoin (manque d'être provisoire) qui, lui, peut être comblé.

Dans cette optique, la mise en relation des sciences, de l'éthique et de la technique qui passerait d'une conception fonctionnaliste à une conception "machinique» serait davantage à l'heure de notre conscience, où le 